\section{Cureus}

\title{
Regression of a Fungating Tumor After Hypofractionated Radiation Therapy in a Patient With Metastatic Breast Cancer
}

\author{
Kevin Quackenbush ${ }^{1}$, Arya Amini ${ }^{1}$, Christine M. Fisher ${ }^{1}$, Rachel Rabinovitch ${ }^{1}$ \\ 1. Department of Radiation Oncology, University of Colorado School of Medicine
}

$\square$ Corresponding author: Kevin Quackenbush, kevin.quackenbush@ucdenver.edu Disclosures can be found in Additional Information at the end of the article

\section{Abstract}

Radiation therapy is a well-established palliative treatment for symptomatic metastases from breast cancer. This is also true of symptomatic primary breast tumors in patients with metastatic disease or in those who are medically inoperable. Further, local progression in the chest wall can severely impair quality of life, with local pain, bleeding, and significant impact on one's self-image. Here, we present the case of a patient who showed an exceptional response to a palliative hypofractionated radiation course to her bleeding, fungating breast primary.

Categories: Radiation Oncology, Oncology

Keywords: breast cancer, palliative radiation, hypofractionated, primary tumor, metastatic breast cancer, locally advanced breast cancer

\section{Introduction}

Breast cancer is the most common cancer in women in the US and the second most common cause of cancer-related death in women. An estimated 252,710 new cases of breast cancer will be diagnosed in the US in 2017, with an estimated 40,610 deaths [1]. An estimated $5 \%-10 \%$ of breast cancer patients have distant metastatic disease at initial presentation, and current guidelines dictate that these patients should receive systemic treatment rather than therapies directed to their primary tumor [1-3]. A recent prospective randomized trial failed to demonstrate an improvement in the overall survival with locoregional treatment of the primary breast tumor in patients presenting with a metastatic disease [4]. However, palliative radiotherapy to the primary breast tumor in the metastatic setting can provide relief from a variety of symptoms including ulceration, bleeding, arm edema, pain, and brachial plexopathy [5].

Received 05/24/2017

\section{Case Presentation}

A 59-year-old woman presented in September 2014 with a left fungating breast tumor. She had been diagnosed two years prior with stage III, ER-/PR-/Her2+ G3 invasive ductal carcinoma and pursued alternative therapies in Mexico including tumor incision "to let the cancer out" as well as intravenous vitamin $C$ therapy. The incision into her breast tumor never closed and she developed a bleeding, painful, malodorous fungating mass. Her staging computed tomography (CT) scan demonstrated metastases to the liver, bones, lungs, and pelvis. Repeat biopsy of the fungating mass revealed poorly differentiated ER+/PR-/Her2+ carcinoma. She was started on systemic treatment with docetaxel, trastuzumab, pertuzumab, and eventually letrozole. She was referred for palliative radiation to the left fungating breast tumor but deferred treatment.

How to cite this article

Quackenbush K, Amini A, Fisher C M, et al. (July 02, 2017) Regression of a Fungating Tumor After Hypofractionated Radiation Therapy in a Patient With Metastatic Breast Cancer. Cureus 9(7): e1417. DOI 10.7759/cureus.1417 


\section{Cureus}

For the next two years, she continued systemic therapy and was eventually switched to adotrastuzumab emtansine and letrozole. She was hospitalized on two separate occasions for acute pain and anemia related to repeat episodes of hemorrhage from her breast tumor. During those hospitalizations, she again deferred palliative radiation treatment. As she continued systemic treatment, her metastatic disease burden remained stable, but her fungating breast tumor had persistent progression.

Due to this worsening local progression, she was referred back to radiation oncology for discussion of treatment. The symptoms stemming from her breast tumor included pain, odor, bleeding requiring repeat transfusions of red cells for blood loss, and emotional distress. Together these were significantly impairing her quality of life, and she opted for palliative radiation. At the time of treatment planning, the patient's left breast tumor measured $10.9 \mathrm{~cm}$ in greatest dimension on the CT scan. A CT-based treatment plan was utilized (Figure 1). Tangential wedged photon fields were placed for planning. No bolus was applied. The setup included weekly electronic portal imaging and given the palliative nature of this treatment, no motion management was performed. She received $35 \mathrm{~Gy}$ in 5 fractions delivered every other day. Ado-trastuzumab emtansine and letrozole were continued during and after radiotherapy. The mean cardiac dose was $0.8 \mathrm{~Gy}$ and mean dose to the left lung was $0.9 \mathrm{~Gy}$. She tolerated her accelerated treatment well and had some minimal tumor regression by the end of her treatment. Her only side effects from the treatment were grade 1 desquamation and grade 1 fatigue, both of which resolved. Over the next several months, her tumor continued to regress and she reported excellent improvement in pain, bleeding, and malodor. Her response continued with near complete regression of the tumor at six months post-treatment (Figures 2-3).

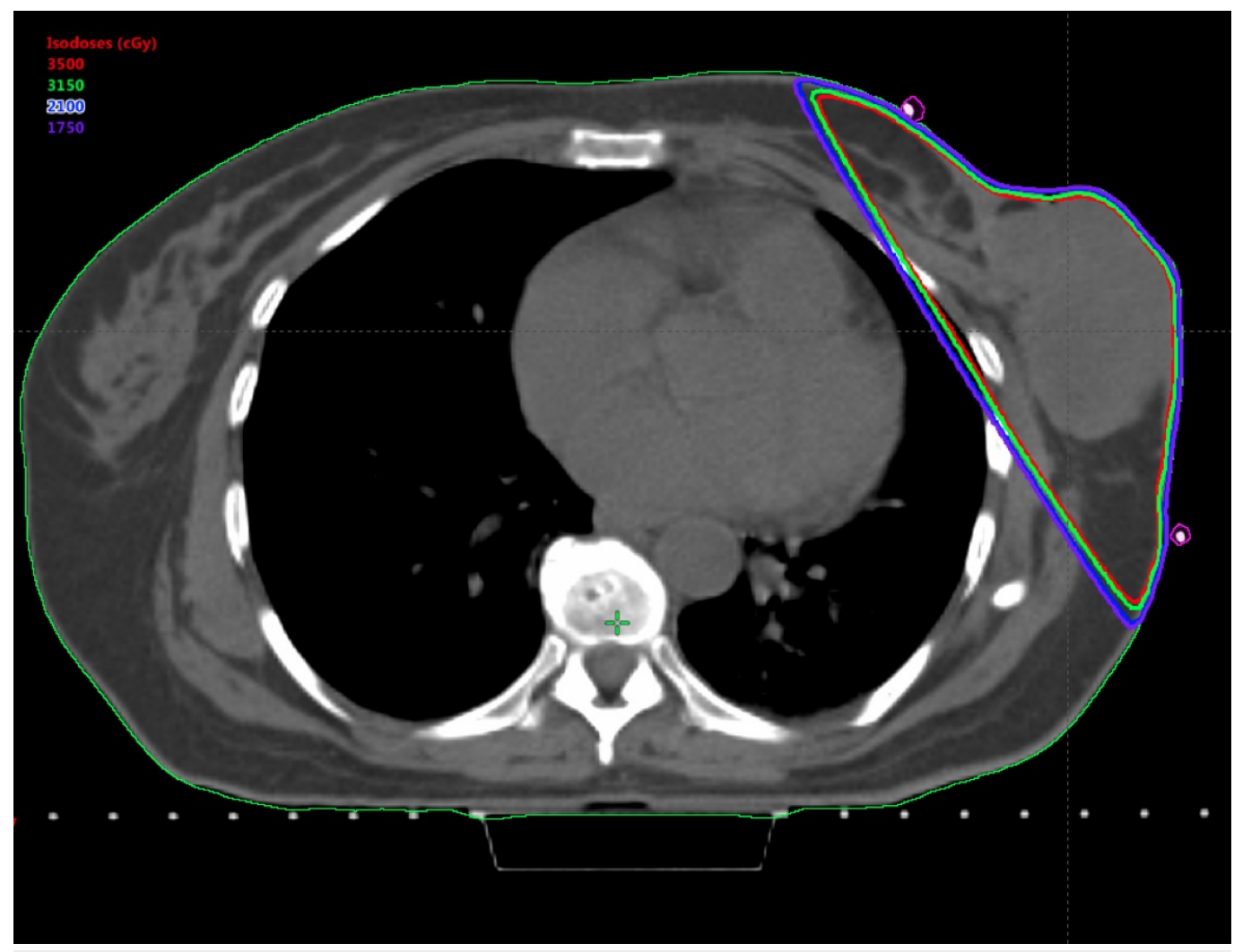

FIGURE 1: Axial image of the radiation treatment plan with representative isodose lines and corresponding doses $(3500$ cGy [red] prescription dose) 


\section{Cureus}

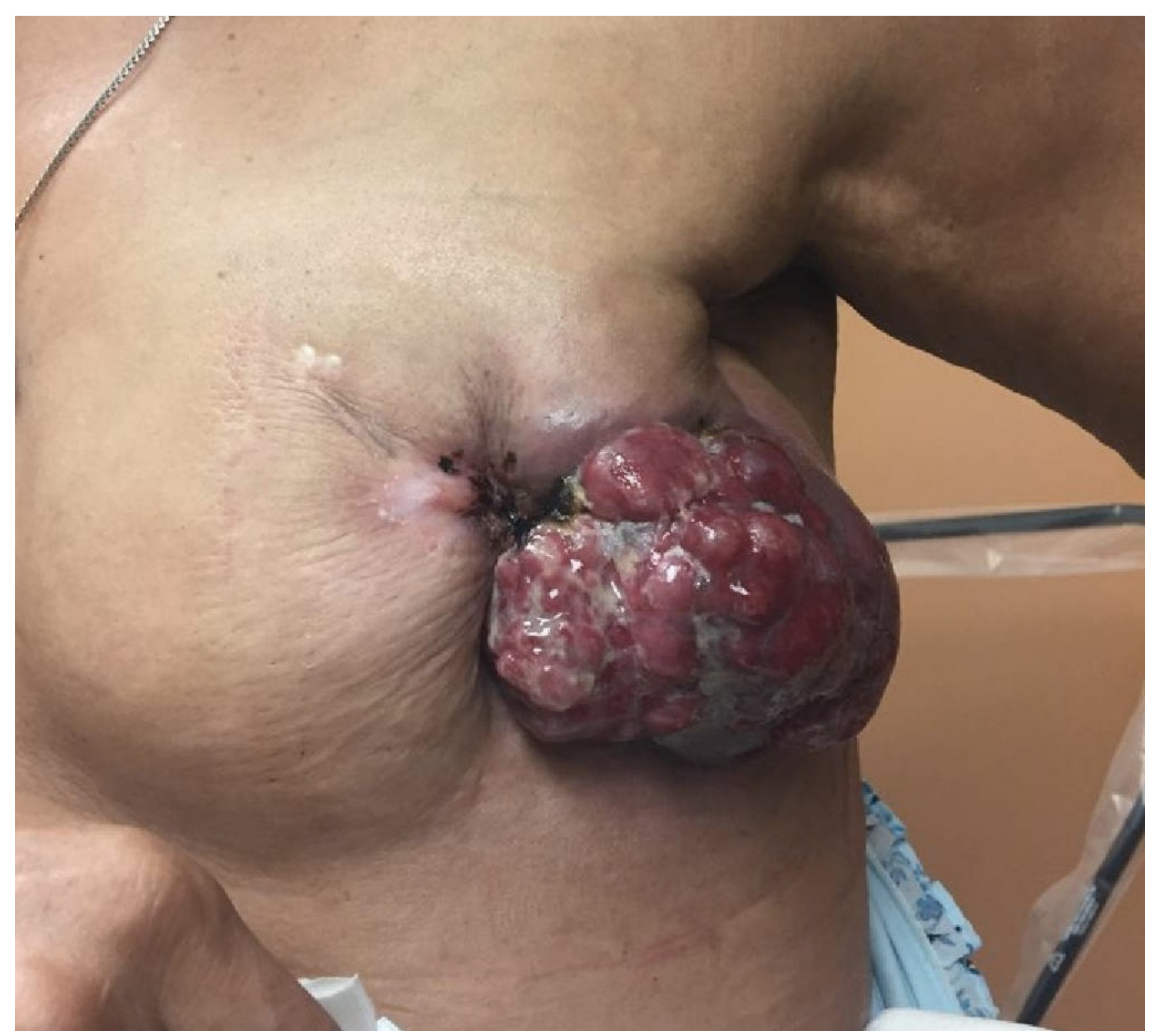

FIGURE 2: Pre-treatment left breast mass 


\section{Cureus}

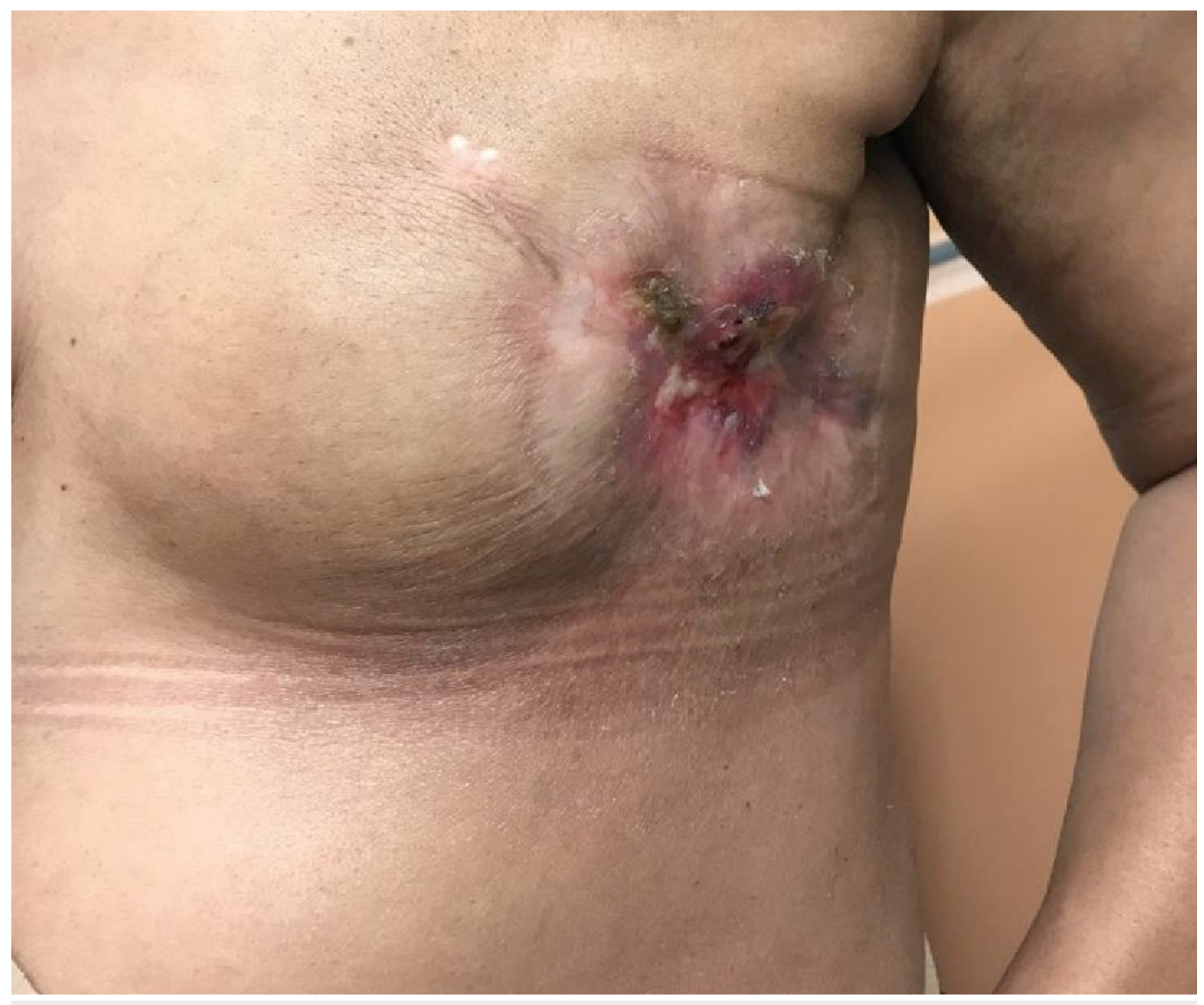

FIGURE 3: Six month post-treatment left breast mass

\section{Discussion}

There is an ongoing debate regarding the benefit of locoregional treatment of the breast with surgery and/or radiation in women whose initial breast cancer presentation includes distant metastatic disease. The question that remains to be answered is what effect locoregional treatment of the primary tumor may have on the overall survival in addition to other measures including patient-reported quality of life. There is retrospective data that has demonstrated a survival benefit to locoregional treatment, but there is concern that these studies have significant selection bias (i.e., younger women and/or women with better performance status may be more likely to be offered locoregional treatment) [3]. While this debate continues, the fact remains that many women with locally advanced or metastatic breast cancer, who have symptoms stemming from their primary breast tumor, can receive tremendous benefit from radiation therapy as a palliative treatment.

There is no guideline-based radiation dose for palliation of a primary breast tumor. Common palliative regimens such as $20 \mathrm{~Gy}$ in 5 fractions or $30 \mathrm{~Gy}$ in 10 fractions may be used in this setting. The benefit of 30-35 Gy in 5 fractions, which is a common regimen offered at our institution, is its higher biologic equivalent dose (BED) compared to other palliative regimens. This dose regimen yields a BED equivalent to $70 \mathrm{~Gy}$ in standard fractionation (2 Gy/fraction), which is a dose appropriate for gross disease in the setting of breast cancer. By comparison, a schedule of $30 \mathrm{~Gy}$ in 10 fractions delivers a BED of only $60 \mathrm{~Gy}$. This, combined with the fact that a $35 \mathrm{~Gy}$ in 5 fraction schedule does not appear to significantly add more side effects and is more convenient for the patient, suggests that the treatment plan used in this case may be an ideal regimen for palliation of a locally advanced breast tumor. 


\section{Conclusions}

Palliative radiation therapy is an effective means of helping to control symptoms such as ulceration, bleeding, and malodor from locally advanced primary breast tumors in the metastatic setting. Longer follow-up is needed to establish the duration of the tumor and symptom control in this setting.

\section{Additional Information Disclosures}

Human subjects: Consent was obtained by all participants in this study. Conflicts of interest: In compliance with the ICMJE uniform disclosure form, all authors declare the following: Payment/services info: All authors have declared that no financial support was received from any organization for the submitted work. Financial relationships: All authors have declared that they have no financial relationships at present or within the previous three years with any organizations that might have an interest in the submitted work. Other relationships: All authors have declared that there are no other relationships or activities that could appear to have influenced the submitted work.

\section{References}

1. Siegel RL, Miller KD, Jemal A: Cancer statistics, 2017. CA Cancer J Clin. 2017, 67:7-30. 10.3322/caac. 21387

2. Gradishar WJ, Anderson BO, Balassanian R, et al.: Invasive breast cancer version 1.2016, NCCN clinical practice guidelines in oncology. J Natl Compr Canc Netw. 2016, 14:324-354.

3. Truong PT: Local treatment of the primary tumor in patients presenting with stage IV breast cancer: a first, and what's up ahead. Int J Radiat Oncol Biol Phys. 2017, 97:443-446.

10.1016/j.ijrobp.2016.08.017

4. Badwe R, Hawaldar R, Nair N, et al.: Locoregional treatment versus no treatment of the primary tumour in metastatic breast cancer: an open-label randomised controlled trial. Lancet Oncol. 2015, 16:1380-1388. 10.1016/S1470-2045(15)00135-7

5. Lutz ST, Jones J, Chow E: Role of radiation therapy in palliative care of the patient with cancer. J Clin Oncol. 2014, 32:2913-2919. 10.1200/JCO.2014.55.1143 